\title{
Modelling the propagation of underwater acoustic emissions for condition monitoring of marine renewable energy
}

\author{
J. Walsh ${ }^{1,2}$, I. Bashir ${ }^{1}$, P. R. Thies ${ }^{1}$, L. Johanning ${ }^{1}$ \\ ${ }^{1}$ College of Engineering, Mathematics and Physical Sciences, University of Exeter, Penryn, UK \\ $\mathrm{Ph}$. Blondel ${ }^{2}$ \\ ${ }^{2}$ Department of Physics, University of Bath, Bath, UK
}

\begin{abstract}
Marine Renewable Energy (MRE) has progressed towards commercialisation over the recent years but significant barriers still exist. This includes the currently high cost of energy, leaving MRE uncompetitive with respect to other more established renewable energy technologies. A significant proportion of this cost comes from Operation and Maintenance (O\&M) activities. O\&M activity can be reduced through the use of condition-based maintenance scheduling. In offshore environments, the submerged location of most devices enables the use of underwater Acoustic Emission (AE), a new condition-monitoring technique. It combines acoustics (used for environmental monitoring of MRE influence on noise levels) with AE condition monitoring as used in air. This paper assesses the practicality of such an approach in complex ocean environments through detailed sound propagation modelling using the propagation model Bellhop in the Matlab toolbox AcTUP. Results show that acoustic propagation is very sensitive to variations in the shallow water environments considered. When concerning sensor placement, multiple-path interferences mean that the location of the measuring sensor(s) needs to be carefully considered, but might not cover all environmental variations over the several months necessary for accurate long-term monitoring. Associated to the shallow depths, these environmental variations also mean that some frequencies cannot be back-propagated easily, generally limiting access to the monitoring of Received Levels. The results presented here are the first steps toward optimizing $\mathrm{AE}$ sensor positions and $\mathrm{AE}$ measuring strategies for arrays of devices.
\end{abstract}

\section{INTRODUCTION}

Over recent years, Marine Renewable Energy (MRE) has progressed towards commercialization but significant barriers still stunt the growth of this developing industry. One such barrier is the overall cost of energy, which is currently significantly higher for MRE than other renewable energy technologies. A substantial proportion of this cost is related to Operation and Maintenance (O\&M) activities. This is due to the MRE devices needing to reside in energetic and hence challenging environments in terms of both the physical accessibility of the site and the durability of the device itself.

It has been shown within the wind energy sector that O\&M costs can be drastically reduced through the use of condition-based maintenance scheduling, where the turbine is monitored for faults through a variety of methods (Mcmillan \& Ault 2007). This is in contrast to the simpler methods of reactive maintenance (performing maintenance when needed) and preventative maintenance (performing maintenance at regular intervals regardless of condition). One such method of monitoring is called Acoustic Emission (AE), currently used in-air within a number of industries (e.g. García Márquez et al. 2012).

This paper explores the practicalities of using underwater AE to monitor the mechanical condition of (partially) submerged MRE devices. The propagation of acoustic signatures observed for MREs within a specific environment is modelled using the open source Matlab Toolbox AcTUP (Maggi \& Duncan n.d.). Section 2 provides an overview of $\mathrm{AE}$ and explores considerations related to underwater AE. Section 3 discusses propagation of underwater AE. Section 4 outlines applications to a Wave Energy Converter in Falmouth Bay (UK) and discusses the relevance of this study to other projects. Finally, a number of conclusions are drawn regarding the level of detail needed to produce helpful modelling results when considering $\mathrm{AE}$ from submerged structures such as MRE devices.

\section{ACOUSTIC EMISSION}

\subsection{Basic principles}

$\mathrm{AE}$ is the sound produced by friction or the release of energy within a material. This potential energy 
could exist due to extra stresses being placed upon the material. In the context of MRE devices, this could be from powerful wave action slamming against the device, mooring lines under tension, or from the general degradation of moving parts from cyclic fatigue.

AE can take the form of signals that are: a) impulsive (distinct acoustic signals, separate in time) or b) continuous signal(s) (impulsive waveforms are not individually distinguishable). Systematic reviews show AE generally occurs at frequencies below 1 $\mathrm{MHz}$, starting at $1 \mathrm{kHz}$ for airborne $\mathrm{AE}$ (Mba \& Rao 2006).

\subsection{Measurements and observations}

Underwater $\mathrm{AE}$ has started to emerge as a viable monitoring method and early publications suggest that useful information can be found at frequencies below $1 \mathrm{kHz}$ (Walsh et al. 2015). Table 1 outlines a recent literature review, which together with measurements in the field has guided laboratory experiments investigating the underwater acoustic emission of breaking of polyester fibre ropes. Impulsive signals across the frequency range $50 \mathrm{~Hz}-48 \mathrm{kHz}$, reaching an amplitude of up to $100 \mathrm{~dB}$ re $1 \mu \mathrm{Pa}$ were detected at a distance of $<1 \mathrm{~m}$ from the source. These levels are conservative as these highamplitude impulsive signals were limited by the instrumentation, sometimes leading to clipped recordings (Bashir et al. 2015).

Other significant results regarding MRE devices have found the power take off (PTO) systems of Wave Energy Converters (WEC) to emit broadband and high amplitude signals when under particularly high stress. Scotrenewables Tidal Power Ltd WEC, Pelamis WEC, Verdant Tidal Turbines and two point absorbers at Lysekil have all reported an increase in emitted noise from devices due to unexpected stresses from anchors, broken blades and incorrect assembly (Walsh et al. 2015).

In particular, in this paper the results of the Fred Olsen's Lifesaver WEC will be used to inform the acoustic propagation modelling inputs. Two years of acoustic data was collected by a hydrophone positioned $200 \mathrm{~m}$ from the WEC location over a broadband of frequencies $(10 \mathrm{~Hz}$ to $32 \mathrm{kHz}$ ) (Garrett 2015). Detailed time-frequency analyses showed that the AE signature of the active PTO system during the WEC operation were 0.5 -second duration bursts up to $90 \mathrm{~dB}$ loud, mostly between $100 \mathrm{~Hz}$ and $1 \mathrm{kHz}$. Loud tonal components at 30, 60, 80 and 100 $\mathrm{Hz}$, reaching $90 \mathrm{~dB}$ were also recorded and attributed to the device generator (Walsh et al. 2016).

Table 1. Summary quality matrix of AE of components relevant to MRE devices including the maximum frequency of the broadband signal detected (Walsh et al. 2015).

Component Fault example Maximum frequency

In air Underwater

\begin{tabular}{|c|c|c|c|}
\hline & & $\mathrm{kHz}$ & $\mathrm{kHz}$ \\
\hline Bearing & Defects on race & 2000 & uninvestigated \\
\hline Gearbox & Gear tooth pitting & 1000 & uninvestigatec \\
\hline Pump & $\begin{array}{l}\text { Cavitation } \\
\text { (incipient and } \\
\text { developed) }\end{array}$ & 20 & 100 \\
\hline \multirow[t]{2}{*}{ Rope } & Fibre fractures & 600 & 48 \\
\hline & Wire fractures & 600 & $100 *$ \\
\hline
\end{tabular}

* In air, through water

\subsection{Ambient noise from the environment}

The ocean is a noisy place, with numerous natural and anthropogenic sources of ambient noise. These sources need to be considered carefully if there is a possibility that underwater $\mathrm{AE}$ monitoring could be used for MRE devices. For example, will an increase in precipitation and sea state mask certain AE? Does a passing ship make the WEC acoustics indistinguishable from background noise? These questions can be addressed with propagation modelling.

Figure 1 shows the Wenz curves, a graphical compilation of the noise present in the ocean from $<$ $1 \mathrm{~Hz}$ to $100 \mathrm{kHz}$ (Wenz 1962). This includes constant sources of sound such as the sea state and molecular agitation, intermittent natural sources, such as biologics and precipitation, and anthropogenic sources including shipping and industrial activity. The graph clearly shows that more ambient noise is concentrated at the lower frequencies, while it is of lesser concern at high frequencies.

\section{REMOTE DETECTION OF ACOUSTIC EMISSION}

\subsection{Underwater sound propagation}

$\mathrm{AE}$ in air is typically performed with sensors located on a component (Mba \& Rao 2006). This does not allow any of the AE to be lost to the propagation through the air. However, underwater this is not as large a con- 


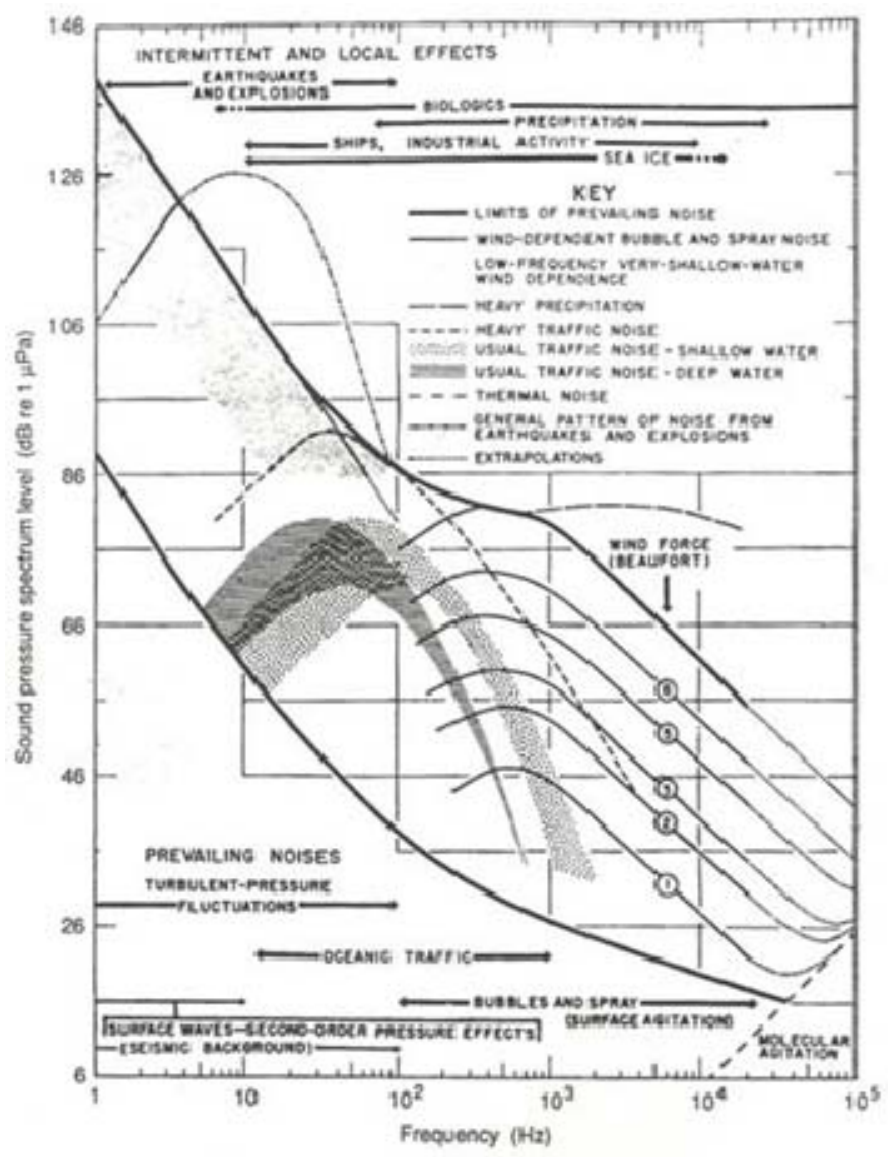

Figure 1. Wenz Curves showing the effects of natural and anthropogenic noise in terms of both power spectral density and frequency within the ocean (Wenz 1962).

cern. The speed of sound in air (at $20{ }^{\circ} \mathrm{C}$ ) is $343 \mathrm{~m} \mathrm{~s}^{-}$ ${ }^{1}$ compared to $1481 \mathrm{~m} \mathrm{~s}^{-1}$ for the speed of sound in (fresh) water (at $20{ }^{\circ} \mathrm{C}$ ). This increase in sound speed underwater enables AE to travel greater distances and still maintain a large proportion of its amplitude, up to several kilometers away in some cases.

Sound propagation in sea water depends on a number of contributing factors of which the most important are temperature, pressure and salinity which together modulate the sound speed profile down the water column. Other factors then include depth, and hence spreading laws to consider, as well as surface boundary roughness, seabed bathymetry and sediment (Etter 2013). Each of these factors may have different influences on different frequencies.

The simplest calculation that can be done to consider the propagation of a sound signal is to calculate the transmission loss (TL) due to spreading. This is often calculated as:

$$
T L=15 \log \left(\frac{R}{R_{\text {ref }}}\right)
$$

where $R$ is the distance from the source to the receiver and $R_{\text {ref }}$ is the reference value of $1 \mathrm{~m}$ (Lurton 2002). This is a compromise between cylindrical and spherical spreading. This calculation is a simple way to estimate propagation losses, but if a frequencydependent answer is needed, or the environment is not homogeneous (e.g. presence of water layers of different salinities or densities), a more rigorous approach is needed.

\subsection{Acoustic Propagation Models}

Rigorous propagation modelling is based upon the wave equation, with the assumption that the force term and pressure are harmonic, leading to the Helmholtz equation,

$$
\nabla^{2} p(\boldsymbol{r})+\frac{\omega}{c(\boldsymbol{r})}(\boldsymbol{r})^{2} p(\boldsymbol{r})=f(\boldsymbol{r})
$$

where $p$ is the pressure, $\mathbf{r}$ is the position, $\omega$ is the angular frequency, $c$ is the speed of sound in the medium and $f$ is the forcing term that represents the acoustic sources (Duncan \& Maggi 2006). There are a number of different models, all with ideal conditions of use that solve this equation with different assumptions. They have all been extensively benchmarked and tested.

The Normal Mode method is a full-field solution that solves the equation for separate horizontal and vertical components. It works best in the far field, in shallow water at low frequencies, however, the fully range dependent model (allowing the input of range dependent variables such as bathymetry) is computationally expensive. The models that use this method include KRAKEN and C-SNAP (Wang et al. 2014).

The Wave Number Integration method is an exact solution at close range only using a numerical approach of spectral wavenumber integration (Wang et al. 2014). This model only publically supports range-independent environments and works best with deep-water, high-frequency problems. The models that use this method include OASES and SAFARI (Wang et al. 2014).

The Parabolic Equation Method is a one-way propagation solution that neglects backscattered rays. It is a range-dependent code, with the ability to support discontinuous sound speed profiles but at frequencies higher than $1 \mathrm{kHz}$ it is computationally extensive This method is coded within RAM and derivative models like RAMGeo (Wang et al. 2014).

Other modelling methods include the Energy Flux Method, a hybrid solution between ray solutions and mode solutions and the Finite Difference/Finite Element methods, a common computational approach to physical problems, but computationally very expensive (Wang et al. 2014). The final method to be mentioned is the Ray Method, used within the model Bellhop, and the model of choice for this paper.

\subsection{Ray-tracing with Bellhop}

The Ray method calculates the path of individual rays coming from the source at different angles and the acoustic field level is calculated by summing up the rays near the receiver (Wang et al. 2014). It allows for range-dependent environments including bathymetry and sea surface, however, the accuracy 
of this model is limited at low frequencies $(<200$ $\mathrm{Hz}$ ), where diffraction and sea bed penetration occur. Interactions of rays with the sea floor are considered via the calculation of a reflection coefficient. The model used to implement the Ray method is Bellhop (Dong \& Dong 2014) in conjunction with Bounce for reflection coefficients (Maggi \& Duncan n.d.).

Bellhop is the propagation model chosen in this paper. This is largely due to its computational efficiency, range-dependent input abilities and seafloor interaction consideration. The reduced accuracy at low frequencies was considered but as there is a large amount of contaminating shipping noise at low frequencies in the area being considered anyway, it was not deemed an issue. Bellhop is also a good choice given its inclusion within AcTUP (Acoustic Toolbox User interface and Post processor - V2.2L) a user-friendly Matlab toolbox that allows for a consistent user interface between multiple propagation models. This includes Bellhop, Bounce, Kraken, and RAM. The interface of multiple models will allow this work to progress easily to a comparison of the models described in Section 3.2 in the context of this work in the future.

\section{COMPARISON OF MODELLING CONDITIONS: RESULTS}

Bounce and Bellhop were used to model the transmission loss that would affect the acoustic emission signal recorded during the testing of the Lifesaver WEC in Falmouth Bay, UK, that was attributed to the PTO. This was briefly described in Section 2.2 and is fully detailed in Walsh et al. 2016. The acoustic signature was broadband $(100 \mathrm{~Hz}-1 \mathrm{kHz})$ with tonal components at 30,60, 80 and $100 \mathrm{~Hz}$. At 200 $\mathrm{m}$ from the source, these signals reached $90 \mathrm{~dB}$ in amplitude. Bellhop however is limited in its abilities for depths less than 20 times the wavelength considered. To ensure meaningful results were obtained, the highest frequency of significance $(1 \mathrm{kHz})$ was used to create these results.

\subsection{Model parameters}

Two propagation models were created, one with idealized conditions of a flat seafloor, flat sea surface and ideal reflector, and the other including a local bathymetry profile and actual sediment information for a more accurate modelling environment.

Parameters varied within the model were the depth of the water column $h$, the speed of sound in the water column $c_{w}$, the density of the water column $d_{w}$, the speed of sound through the seafloor $c_{f}$, and the density of the seafloor $d_{f}$. Table 2 outlines the different parameters used for both the idealized and actual models.
The sound speed profile of the water column was kept at a constant value $\left(1,500 \mathrm{~m} \mathrm{~s}^{-1}\right)$ due to the small variation seen during the winter months due to increased mixing from the weather (Garrett 2015). For this reason also, the density was kept constant. However, the depth of the water column changed between models. The idealized model kept a flat seafloor $40 \mathrm{~m}$ deep whereas the actual model included a simple slope to represent the most extreme bathymetry seen in the area (Garrett 2015).

The seafloor itself was given the default values from AcTUP for the idealized conditions. For the more detailed conditions, a sediment sound speed was used (Etter 2013), with a density of wet, packed, sand (The Engineering Toolbox n.d.).

The model input frequency was $1,000 \mathrm{~Hz}$. The source was placed at reference range of $0 \mathrm{~m}$ and $5 \mathrm{~m}$ below the sea surface to mimic the submerged area of the WEC from where the sound would propagate.

\subsection{Results}

These parameters were used for 2 Bounce and Bellhop configurations, one for idealized conditions, and one for a more accurate consideration of the local environment. Figure 2 shows the results for the idealized conditions, the orange line showing the associated model bathymetry. This plot of Transmission Loss (in $\mathrm{dB}$ ) as a function of range and depth shows how the rays of the model interfere throughout the associated area to produce areas of concentrated TL and areas of very small TL. Figure 3 shows the same plot for the more realistic

Table 2. The significant environment parameters used within the idealized and detailed Bounce and Bellhop models within AcTUP.

\begin{tabular}{lccccccc}
\hline Model & \multicolumn{3}{l}{ Water column } & & \multicolumn{2}{l}{ Seafloor } \\
\cline { 2 - 4 } \cline { 6 - 7 } & $h$ & $c_{w}$ & $d_{w}$ & & $c_{f}$ & $d_{f}$ \\
\cline { 2 - 4 } \cline { 6 - 7 } & $\mathrm{m}$ & $\mathrm{m} \mathrm{s}^{-1}$ & $\mathrm{~kg} \mathrm{~m}^{-3}$ & & $\mathrm{~m} \mathrm{~s}^{-1}$ & $\mathrm{~kg} \mathrm{~m}^{-3}$ \\
\hline Idealized & 40 & 1500 & 1024 & & 1749 & 1941 \\
Detailed & $40-60$ & 1500 & 1024 & & 2000 & 2082 \\
\hline
\end{tabular}

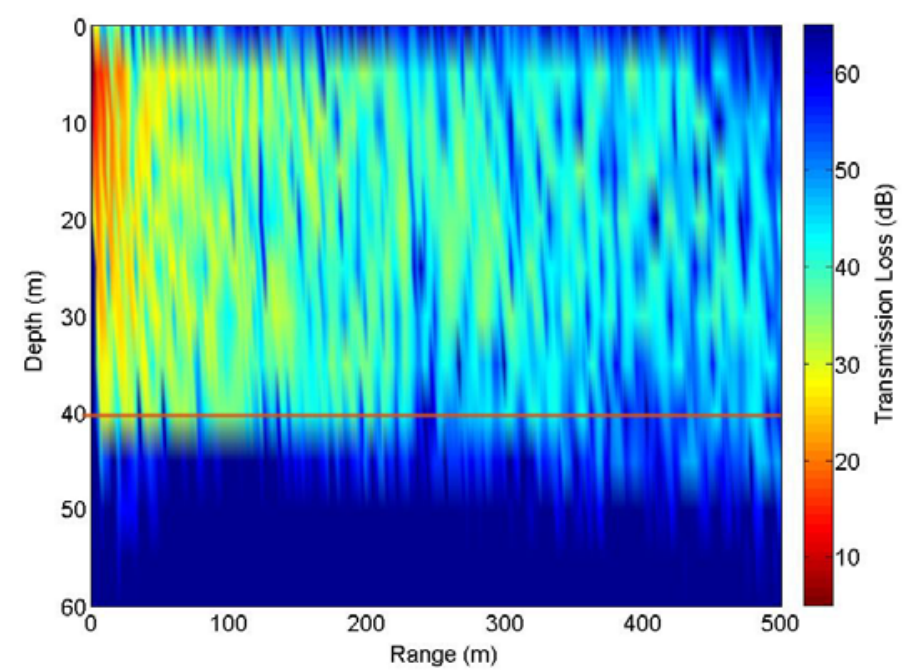

Figure 2. Transmission Loss (in $\mathrm{dB}$ ) modelled as a function of range and depth for an idealized environment with Bounce and 
Bellhop. The orange line shows the model bathymetry; the AE source was placed at $5 \mathrm{~m}$ depth and $0 \mathrm{~m}$ range.

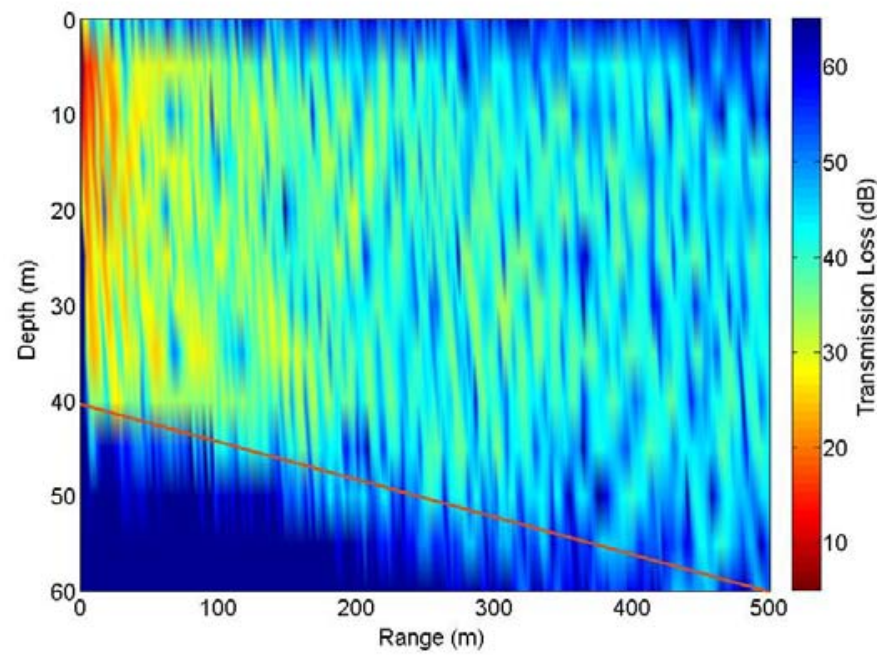

Figure 3. Transmission Loss (in $\mathrm{dB}$ ) modelled as a function of range and depth for a more realistic and detailed environment with Bounce and Bellhop. The orange line shows the model bathymetry; the $\mathrm{AE}$ source was placed at $5 \mathrm{~m}$ depth, at $0 \mathrm{~m}$ range.
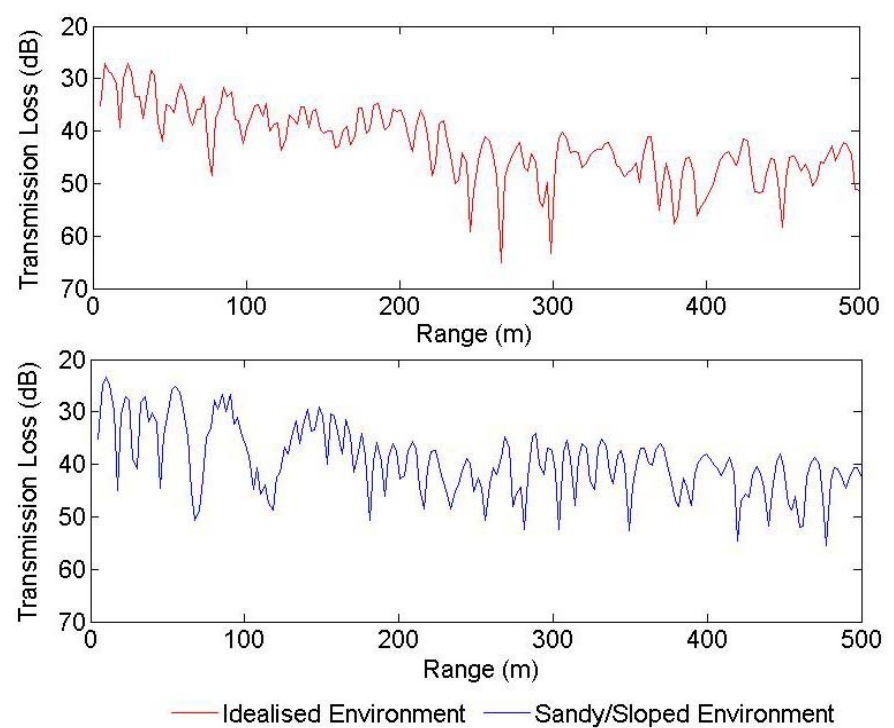

Figure 4. Comparison of the Transmission Loss (in $\mathrm{dB}$ ) for a $1,000 \mathrm{~Hz}$ signal through idealized and detailed propagation environments at source depth of $5 \mathrm{~m}$ and a receiver depth of $35 \mathrm{~m}$ (i.e. close to the seabed).

and detailed model, showing again in orange the sloping seafloor used to approximate the local bathymetry. Predictions using Equation 1 at $R=200 \mathrm{~m}$ (where the field measurements were made) yield: $T L$ $=46 \mathrm{~dB}$.

\section{DISCUSSION}

While Figure 2 and 3 provide a good aid to visualizing the sound field in the area and water column considered, it is difficult to understand the variability of the transmission loss. Figure 4 however, shows the transmission loss at $35 \mathrm{~m}$ depth (the approximate depth of the receiver in the associated study) for both the idealized and detailed propagation models.
Figure 4 clearly shows that multipath propagation in such a shallow environment creates huge variability in the transmission loss that is especially sensitive to position (range) as well as between input model parameters. The movement of the receiver by just a few meters through the range could create variations in the transmission loss of up to $20 \mathrm{~dB}$. This variability is much higher than the expected experimental uncertainty of $\pm 1 \mathrm{~dB}$ (Robinson et al. 2014) and has a number of consequences.

Firstly, when such large variations exist, the ability to use back-propagation to calculate a source's effective Source Level is near impossible. This has recently been considered in detail within the specific domain of Environmental Impact Assessments (Farcas et al. 2016), with similar conclusions. Received Levels, if appropriately documented (Merchant et al. 2015; Robinson et al. 2014), should however be most useful when comparing different MRE devices.

Secondly, from a more practical point of view, the marine environment is extremely dynamic, meaning that both source and receiver will move around their assumed positions. Wave Energy Converters will move with winds, waves, currents and forces acting on their moorings to the seabed. Acoustic receivers will move due to underwater currents. Both types of influences can create variations in known relative positions of the source and receiver of tens of meters. This in turn will impact upon any propagation model results with variations of up to $20 \mathrm{~dB}$ in transmission loss.

Finally, Figure 4 shows that at $200 \mathrm{~m}$ range, there is a $5 \mathrm{~dB}$ difference between the two input model parameters. This highlights the importance of using the most accurate environment description, along with the exact frequencies to be considered. Model appropriateness is further discussed across multiple publications, e.g. Farcas et al. 2016; Wang et al. 2014; Etter 2013.

Another significant result from Figure 4 highlights that there are significant areas where a large Transmission Loss is accumulated over a significant range. For example, in Figure 4, between $100 \mathrm{~m}$ and $125 \mathrm{~m}$, there is a sustained transmission loss of $45-$ $50 \mathrm{~dB}$. Information such as this could inform the position of acoustic receivers when a specific frequency or signal is of importance, such as tonal noises from generators. These are generally low-frequency signals, and would need more modelling with a more appropriate model to confirm these "quiet zones" exist at lower frequencies.

Other further work could compare the results of multiple well-used models within the field such as Kraken, Ram and Bellhop in the context of mediumto high-frequency AE signals. The influence of sea state, sound speed profiles and shipping will also need to be investigated. 
Further work specific to the Lifesaver data will look to compare the transmission loss across the known bathymetry paths of the multiple hydrophone deployments of Garrett 2015. Considering the difference in Transmission Loss across multiple bathymetry profiles will provide a deeper understanding of the differences between deployments.

\section{CONCLUSION}

For MRE to become a competitive industry in the renewable energy market, the cost of energy must be reduced. This can be achieved via condition-based monitoring using techniques such as underwater $\mathrm{AE}$ to monitor the health of an MRE device. Underwater acoustic propagation modelling can be used to better understand the effect of a device on the local soundscape. This can help to develop the practicalities of sensor location, numbers and even array design.

This paper has shown the huge variability $( \pm 20$ $\mathrm{dB}$ ) shown by the propagation model Bellhop. With such variability, back-propagation to actual Source Levels becomes highly inaccurate and Received Levels, properly documented, are the best way forward. This variability in shallow water is sensitive to environmental parameters such as bathymetry and sediment properties. Further work will compare with other propagation models and further develop the modelling associated with the Lifesaver deployment.

\section{ACKNOWLEDGEMENTS}

JW is funded by the Natural Environment Research Council (NERC grant NE/L002434/1) as part of the GW4+ Doctoral Training Partnership (http://www.nercgw4plus.ac.uk/). IB is funded through the SuperGen UK Centre for Marine Energy Research (EPSRC grant EP/M014738/1).

\section{REFERENCES}

Bashir, I. et al., 2015. Underwater acoustic emission testing of mooring ropes at Dynamic Marine Component Test Facility (DMaC). In 2nd Annual PRIMaRE Conference (Abstract). Penryn, UK, p. 1.

Dong, L. \& Dong, H., 2014. Bellhop - A modeling approach to Sound propagation in the ocean. In Proceedings of the 36th Scandinavian Symposium on Physical Acoustics. Geilo, Norway, pp. 1-4.

Duncan, A.J. \& Maggi, A.L., 2006. A consistent, user friendly interface for running a variety of underwater acoustic propagation codes. In Proceedings of Acoustics 2006. Christchurch, New Zealand, pp. 471-477.

Etter, P.C., 2013. Underwater Acoustic Modeling and Simulation Fourth., CRC Press.

Farcas, A., Thompson, P.M. \& Merchant, N.D., 2016. Underwater noise modelling for environmental impact assessment. Environmental Impact Assessment Review, 57, pp.114-122.

García Márquez, F.P. et al., 2012. Condition monitoring of wind turbines: Techniques and methods. Renewable Energy, 46, pp.169-178.

Garrett, J.K., 2015. Interdisciplinary study into the effect of a marine renewable energy testing facility on the underwater sound in Falmouth Bay. University of Exeter.

Lurton, X., 2002. An introduction to underwater acoustics: Principles and applications, Springer.

Maggi, A. \& Duncan, A., AcTUP - installation and user guide, Available at: http://cmst.curtin.edu.au/products/actool box.cfm.

Mba, D. \& Rao, R., 2006. Development of acoustic emission technology for condition monitoring and diagnosis of rotating machines; bearings, pumps, gearboxes, engines and rotating structures. The shock and vibration digest, 38(1), pp.3-16.

Mcmillan, D. \& Ault, G.W., 2007. Quantification of condition monitoring benefit for offshore wind turbines. Wind Engineering, 31(4), pp.267-285.

Merchant, N.D. et al., 2015. Measuring acoustic habitats. Methods in Ecology and Evolution, 6(3), pp.257-265.

Robinson, S.P., Lepper, P.A. \& Hazelwood, R.A., 2014. Good Practice Guide for Underwater Noise Measurement. National Measurement Office, Marine Scotland, The Crown Estate. Good Practice Guide No. 133,

The Engineering Toolbox, Densities of some common materials. Available at: http://www.engineeringtoolbox.com/density -materialsd_1652.html [Accessed February 26, 2016].

Walsh, J. et al., 2015. Acoustic Emission Health Monitoring of Marine Renewables Illustration with a Wave Energy Converter in Falmouth Bay ( UK ). In MTS/IEEE Oceans15. Genova, Italy:

Walsh, J. et al., 2016. Monitoring the condition of Marine Renewable Energy Devices through underwater Acoustic 2 Emissions: Case study of a Wave Energy Converter in Falmouth Bay, UK. Submitted to Renewable Energy, p.15.

Wang, L.S. et al., 2014. Review of underwater acoustic propagation models, National Physical Laboratory.

Wenz, G.M., 1962. Acoustic Ambient Noise in the Ocean: Spectra and Sources. The Journal of the Acoustical Society of America, 34(12), p.1936. 
